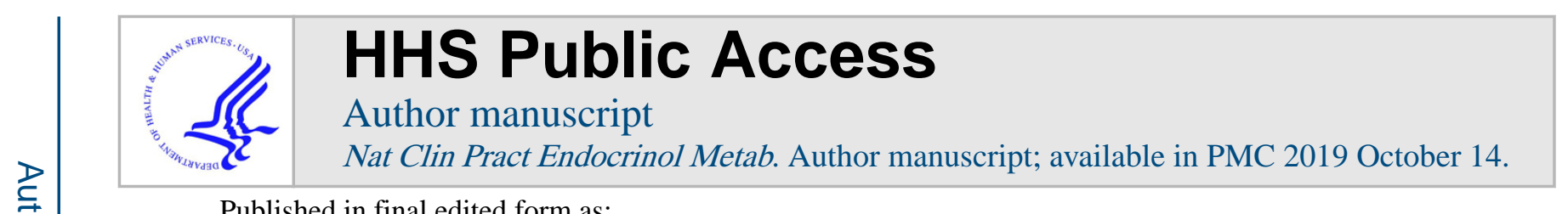

Published in final edited form as:

Nat Clin Pract Endocrinol Metab. 2006 November ; 2(11): 592-593. doi:10.1038/ncpendmet0320.

\title{
Management of obesity in Prader-Willi syndrome
}

\author{
Merlin G Butler \\ MG Butler is the William R Brown/Missouri Chair in Medical Genetics, Chief of the Section of \\ Medical Genetics and Molecular Medicine, Children's Mercy Hospitals and Clinics, and Professor \\ of Pediatrics, University of Missouri-Kansas City School of Medicine, Kansas City, MO, USA.
}

Prader-Willi syndrome (PWS) is considered the most common genetic cause of morbid obesity and has a birth incidence of 1 in 25,000-30,000. ${ }^{1,2}$ PWS is characterized by infantile hypotonia, failure to thrive, hypogonadism, small hands and feet, short stature, intellectual challenges, behavioral problems, food foraging, and hyperphagia leading to early childhood obesity. ${ }^{1-3}$ Gastric rupture is also a reported complication in PWS. ${ }^{3}$ Individuals with PWS often have a significant preoccupation with food, experience constant hunger, and display food-seeking behavior. During the first stage of PWS (onset at birth), poor suckling and feeding difficulties require specific dietary intervention for weight gain; however, during the second stage (onset 2-4 years of age) an insatiable appetite develops, which is followed by rapid weight gain. Without intervention, obesity occurs, and this is the most significant health problem of PWS—obesity and its complications can potentially be life-threatening. In this Viewpoint, I will discuss strategies to treat obesity in this complex disorder, outline medical and surgical options, then concentrate on nutritional and behavioral management of energy expenditure.

Energy expenditure components are estimated by a variety of methods but include resting metabolism (accounting for $\sim 60 \%$ of energy expenditure), thermic effects of food (energy required to consume and digest food; $\sim 10 \%$ of energy expenditure), and physical activity (spontaneous or planned exercise). Growth hormone deficiency is recognized as a key component of PWS and replacement therapy at a young age can have beneficial effects on height and body composition by expanding lean mass. The increase in lean mass raises the metabolic rate and increases physical activity and energy expenditure, which leads to a decreased fat mass and weight loss. Similar improvements in body composition and quality of life have been noted in adults with PWS who were treated with growth hormone. ${ }^{4}$

Success with pharmacologic agents for weight management in PWS has so far been limited. None of the appetite suppressants (e.g. sibutramine) or antiabsorptive agents (e.g. orlistat) marketed for obesity treatment has been systematically studied in PWS. Decreased appetite and reduced weight have been observed in patients with PWS who use topiramate (an antiepileptic agent) and metformin (an oral antidiabetic agent), but many of the data are

Correspondence: Section of Medical Genetics and Molecular Medicine, Children's Mercy Hospitals and Clinics, 2401 Gillham Road, Kansas City, MO 64108, USA, mgbutler@cmh.edu.

Competing interests

The author declared he has no competing interests. 
anecdotal. Indeed, a report by Shapira et al..$^{5}$ demonstrated a lack of benefit of topiramate in terms of appetite and obesity in PWS.

Ghrelin and peptide YY (PYY) are small peptides produced by the gastrointestinal organs, which are involved in appetite regulation (ghrelin stimulates eating, whereas PYY inhibits eating). ${ }^{3,6}$ Interestingly, elevated fasting plasma ghrelin levels have been reported in infants, children, and adults with PWS. ${ }^{3}$ Ghrelin-antagonists and PYY agonists are, therefore, potentially new classes of obesity drugs that might have a role in the treatment of PWS. In preliminary studies, Tan et al. ${ }^{7}$ found that acute reduction of ghrelin levels by infusion of somatostatin in adults with PWS did not reduce food intake. Studies are underway to investigate the possible benefit of chronic administration of somatostatin analogs to correct hyperghrelinemia in PWS. In addition, rimonabant, an experimental drug that could possibly combat both obesity and smoking, might also have treatment potential in PWS.

Surgery is an alternative approach to managing PWS, but because of hyperphagia and a concern for gastric rupturing, surgically decreasing the stomach volume could pose unwanted and life-threatening risks. Because of a paucity of data regarding bariatric surgery in PWS it should, therefore, be considered only in severe cases where serious obesity-related comorbidities are present, and rapid weight loss is needed. ${ }^{3}$

Strategies that involve the patient, family members, and care providers are critical for the development of ways to cope with the weight and behavior control issues characteristic of PWS ${ }^{8}$ and patients require close supervision at all times. Tactics include locking away food, keeping limited amounts of food in the home, continual close supervision of the patient around food or food-related events, providing non-food-related rewards, reduction of portion sizes with small plates and bowls, allowing participation in menu planning and preparation, counting calories, and having access to food with fewer calories. It is also important to keep strict mealtime regimes, and to ensure unwavering consistency by both parents and care providers, inside and outside of the home. Individually tailored exercise programs are encouraged, and 30 min of sustained activity $3-5$ times per week is generally recommended.

The overall goal of nutritional management of PWS is to provide optimal nutrition for health and growth, achieve weight control, and preserve (or increase) lean body mass. Compared with the general population, the typical PWS body composition includes increased fat mass and decreased muscle mass and bone density. The standard balanced caloric plan in general use (60\% carbohydrate, $15 \%$ protein and $25 \%$ fat) requires modification in patients with PWS. A possible approach might be to supply a third of calories from protein, two-fifths from carbohydrate, and the remainder from fat. ${ }^{3}$ Provision of adequate protein during times of caloric restriction should help conserve lean body mass. The American Dietetic Association food exchange programs (which allow the substitution of starch or bread, vegetables, fruit, meat, dairy products, and fats for each other) and/or the US Department of Agriculture food guide pyramid can be helpful to select food sources containing protein, carbohydrates, and fats to ensure that the nutritional needs (in terms of both weight management and growth) of children and adults with PWS are met. The regime requires regular dietary counseling of care providers and includes the analysis of food records, as 
well as attention to individual nutritional deficiencies. It is equally important to relate weight loss to body composition changes with dual energy X-ray absorptiometry.

To control energy intake, a careful food monitoring program is essential for both children and adults with PWS. Food intake during the first 2 years of life requires adjustment to maintain weight for height measures between the $25^{\text {th }}$ and $80^{\text {th }}$ percentile. Published growth standards for PWS are available for this purpose. ${ }^{9}$ Restricted caloric intake with vitamin and calcium supplementation performed under the close supervision of an experienced dietitian is generally required by age $2-3$ years and beyond to minimize excessive weight gain and osteoporosis. Historically, weight maintenance in children with PWS who have not been treated with growth hormone has been reported with an intake of 8-11 kcal per $\mathrm{cm}$ of height per day, whereas children without PWS require 11-14 kcal per $\mathrm{cm}$ per day for adequate growth. ${ }^{10}$ Anecdotal reports indicate that growth hormone therapy could have a beneficial effect on eating behavior but no objective studies have been performed. Additionally, weight loss has been documented in children with PWS by restricting their caloric intake to $7 \mathrm{kcal}$ per $\mathrm{cm}$ per day. Similar guidelines have not yet been developed specifically for adolescents and adults (males or females) with PWS, although 800-1,000 kcal daily is the limit generally needed for weight loss.

In conclusion, energy expenditure and obesity can be controlled in patients with PWS, but a lifetime commitment and close observation are required by all involved in the care of affected individuals.

\section{References}

1. Bittel DC and Butler MG (2005) Prader-Willi syndrome: clinical genetics, cytogenetics and molecular biology. Expert Rev Mol Med 7: 1-20

2. Whittington JE et al. (2001) Population prevalence and estimated birth incidence and mortality rate for people with Prader-Willi syndrome in one UK health region. J Med Genet 38: 792-798 [PubMed: 11732491]

3. Scheimann AO et al. (2006) Gastrointestinal system, obesity and body composition In Management of Prader-Willi Syndrome, 153-200 (Eds Butler MGet al.) New York: Springer-Verlag

4. Carrel AL et al. (2006) Growth hormone and Prader-Willi syndrome In Management of PraderWilli Syndrome, 201-241 (Eds Butler MGet al.) New York: Springer-Verlag

5. Shapira NA et al. (2004) Effects of topiramate in adults with Prader-Willi syndrome. Am J Ment Retard 109: 301-309 [PubMed: 15176917]

6. Stanley S et al. (2005) Hormonal regulation of food intake. Physiol Rev 85: 1131-1158 [PubMed: 16183909]

7. Tan TM et al. (2004) Somatostatin infusion lowers plasma ghrelin without reducing appetite in adults with Prader-Willi syndrome. J Clin Endocrinol Metab 89: 4162-4165 [PubMed: 15292365]

8. Goldberg D et al. (2002) Coping with Prader-Willi syndrome. J Am Diet Assoc 102:537-542 [PubMed: 11985412]

9. Butler MG and Meaney FJ (1991) Standards for selected anthropometric measurements in PraderWilli syndrome. Pediatrics 88: 853-860 [PubMed: 1896298]

10. Holm VA and Pipes PL (1976) Food and children with Prader-Willi syndrome. Am J Dis Child 130: 1063-1067 [PubMed: 973608] 